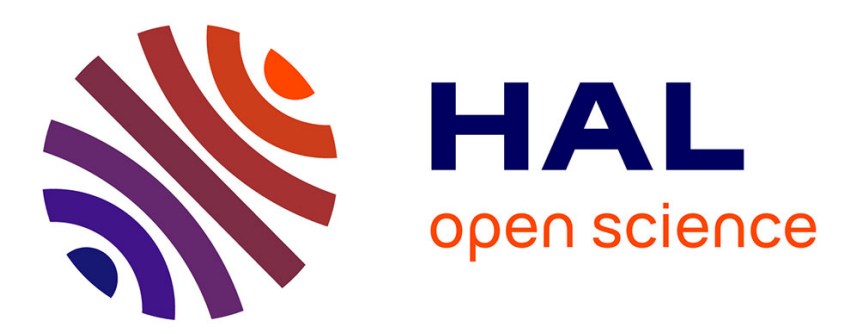

\title{
Paramètres de transport et lois d'échelle dans les solutions diluées de polymères flexibles \\ Anne Schmitt
}

\section{To cite this version:}

Anne Schmitt. Paramètres de transport et lois d'échelle dans les solutions diluées de polymères flexibles. Journal de Physique Lettres, 1979, 40 (13), pp.317-318. 10.1051/jphyslet:019790040013031700 . jpa-00231633

\section{HAL Id: jpa-00231633 https://hal.science/jpa-00231633}

Submitted on 1 Jan 1979

HAL is a multi-disciplinary open access archive for the deposit and dissemination of scientific research documents, whether they are published or not. The documents may come from teaching and research institutions in France or abroad, or from public or private research centers.
L'archive ouverte pluridisciplinaire HAL, est destinée au dépôt et à la diffusion de documents scientifiques de niveau recherche, publiés ou non, émanant des établissements d'enseignement et de recherche français ou étrangers, des laboratoires publics ou privés. 


\title{
Paramètres de transport et lois d'échelle dans les solutions diluées de polymères flexibles
}

\author{
A. Schmitt \\ C.N.R.S., Centre de Recherches sur les Macromolécules, 6, rue Boussingault, 67083 Strasbourg Cedex, France
}

(Reçu le 12 mars 1979, révisé le 4 mai 1979, accepté le 8 mai 1979)

\begin{abstract}
Résumé. - Pour un polymère flexible en solution diluée, nous montrons qu'il est possible, à partir de mesures de diffusion et de viscosité, d'étudier les variations du rayon de giration $R_{\mathrm{G}}$ avec le nombre de monomères $N$. On met ainsi en évidence le phénomène de crossover au voisinage d'un nombre critique $N_{\mathrm{c}}$, et les lois de variation $R_{\mathrm{G}} \propto N^{v}$ prévues théoriquement pour $N \gtrless N_{\mathrm{c}}$.
\end{abstract}

\begin{abstract}
We show that, by combining diffusion and viscosity measurements for a flexible polymer in the dilute regime, it is possible to study the variation of the radius of gyration $R_{\mathrm{G}}$ with the number of monomers $N$. The crossover phenomenon is observed around a critical value $N_{\mathrm{c}}$, together with the expected power laws $R_{\mathrm{G}} \propto N^{v}$ for $N \gtrless N_{\mathrm{c}}$.
\end{abstract}

La détermination, en solution diluée, des dimensions de polymères flexibles par le biais de paramètres de transport (coefficients de diffusion, de sédimentation, viscosité intrinsèque) conduit, en bon solvant, à des difficultés d'interprétation $[1,2]$. En effet, les lois de puissance traduisant la variation de ces paramètres avec le degré de polymérisation $N$ ne sont pas en accord avec les prédictions théoriques, contrairement aux données résultant de mesures statiques [3].

Pour rendre compte de ces faits expérimentaux, l'hypothèse d'indices dynamiques a été évoquée $[2,4]$. Toutefois, dans un article récent [5], Weill et Des Cloizeaux ont proposé une interprétation qui paraît beaucoup plus convaincante. En introduisant deux distances caractéristiques $R_{\mathrm{G}}$ (rayon de giration) et $R_{\mathrm{D}}$ (rayon de diffusion), il est montré que la viscosité intrinsèque $[\eta]$ et le coefficient de diffusion $D$ s'expriment par les relations :

$$
\begin{aligned}
{[\eta] } & \propto \frac{R_{\mathrm{G}}^{2} R_{\mathrm{D}}}{N M_{\mathrm{m}}} \\
D & \propto R_{\mathrm{D}}^{-1}
\end{aligned}
$$

où $M_{\mathrm{m}}$ est la masse d'un monomère. Cependant, dans le domaine des masses moléculaires habituellement exploré, $R_{\mathrm{G}}$ atteint pratiquement sa valeur asymptotique alors qu'il n'en est pas ainsi pour $R_{\mathrm{D}}$. Dans la définition de la distance moyenne $R_{\mathrm{D}}$, il est clair que le poids statistique des petites distances est important, ce qui reporte la limite asymptotique aux très fortes valeurs de $N$. En considérant un modèle de chaîne polymérique en bon solvant, Weill et Des Cloizeaux ont montré que l'on pouvait rendre compte de manière satisfaisante des indices dynamiques observés.

Il est possible de corroborer l'interprétation brièvement résumée à partir de la remarque suivante. En effectuant le produit $[\eta] D$, il apparaît d'après (1) et (2) que la distance gênante $R_{\mathrm{D}}$ s'élimine,

$$
[\eta] D \propto \frac{R_{\mathrm{G}}^{2}}{N M_{\mathrm{m}}}
$$

et l'on peut ainsi étudier la variation du rayon de giration avec $N$.

Utilisant des résultats publiés par Mukherjea et Rempp [6] et complétés par Freund [7] (voir tableau I) nous avons porté sur la figure 1 les variations du paramètre $[\eta] D$ avec le degré de polymérisation, en coordonnées logarithmiques, pour des fractions de polystyrène assez monodisperses, en solution dans du toluène à $20^{\circ} \mathrm{C}$. Pour $N>100$, on observe une pente $p$ voisine de 0,2 . D'après les lois d'échelle [2], $p$ est relié à l'indice critique $v$ par la relation :

$$
p=2 v-1 \text {. }
$$

Ceci conduit à la valeur $v \simeq 0,6$, très voisine de celle théoriquement attendue. En utilisant des lois de puissance récemment obtenues pour le système poly- 
Tableau I. - Variations du coefficient de diffusion $D$ et de la viscosité intrinsèque [ $\eta]$ de fractions assez monodisperses de polystyrène, de degré de polymérisation moyen $N$, en solution diluée dans le toluène à $20^{\circ} \mathrm{C}$. Notons que le point expérimental correspondant à la fraction marquée $\left(^{*}\right)$ n'est pas représenté sur la figure 1 , car il paraît aberrant.

[Variations of the diffusion coefficient $D$ and the intrinsic viscosity $[\eta]$ of relatively monodisperse polystyrene fractions, of mean degree of polymerization $N$, in dilute toluene solutions at $20^{\circ} \mathrm{C}$. Note that the experimental point related to the fraction marked $\left(^{*}\right)$ is not represented in figure 1 , for it appears erroneous.]

$\begin{array}{ccc}N & \begin{array}{c}10^{7} \times D \\ \left(\mathrm{~cm}^{2} \mathrm{~s}^{-1}\right)\end{array} & \begin{array}{c}{[\eta]} \\ \left(\mathrm{cm}^{3} \mathrm{~g}^{-1}\right)\end{array} \\ 38,5 & - & - \\ 85,6 & 22,6 & 5,85 \\ 206,7 & 14,6 & 9,2 \\ 408,6 & 10,2 & 14,5 \\ 1490 & 7,7 & 22,8 \\ 2519 & 4,0 & 57 \\ 4134\left(^{*}\right) & 3,05 & 80,2 \\ 18150 & 2,02 & 120 \\ 86500 & 1,05 & 388 \\ & 0,66 & 867\end{array}$

styrène-benzène $[8]\left([\eta] \propto N^{0,725} ; D \propto N^{-0,55}\right)$, on obtient aussi, en effectuant le produit $[\eta] D$, la valeur $v \simeq 0,59$.

Un autre aspect intéressant du diagramme 1 est qu'il semble permettre une étude du phénomène de crossover. Celui-ci peut être observé sur une chaîne de grande masse, en étudiant sa diffusion à des échelles différentes [9]. Une méthode alternative consiste à mesurer les variations du rayon de giration avec $N$ sur des chaînes différentes. La figure 1 suggère que la longueur critique de crossover $N_{\mathrm{c}}$ est de l'ordre de 100 , et que pour $N<N_{\mathrm{c}}$, les chaînes sont gaussiennes $(p=0 ; v=0,5)$, conformément aux prévisions théoriques [2]. Il est clair que la valeur de $N_{\mathrm{c}}$ dépend du couple polymère-solvant considéré, ainsi

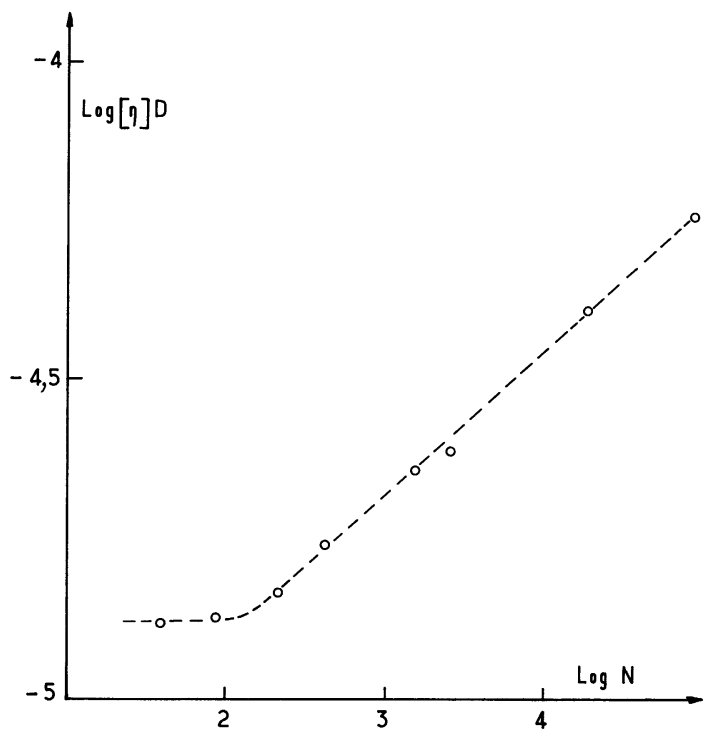

Fig. 1. - Variation du paramètre de transport $[\eta] D$ en fonction du nombre de monomères $N$, en coordonnées logarithmiques, pour le système polystyrène-toluène à $20^{\circ} \mathrm{C}$.

[Variation of the $[\eta] D$ transport parameter as a function of the degree of polymerization $N$, on logarithmic scales, for the polystyrene-toluene system at $20^{\circ} \mathrm{C}$.]

que de la température, comme l'a montré le travail récent de Farnoux et al. [10].

De telles conclusions méritent d'être étayées par des données expérimentales plus fournies. Elles soulignent néanmoins l'intérêt potentiel de pareille approche expérimentale, qui devrait aussi conduire à une estimation plus appropriée du gonflement des chaînes polymériques. Par ailleurs, en chromatographie par perméation de gel, il serait intéressant d'étudier dans quelle mesure une représentation du type $\log N M_{\mathrm{m}}[\eta] D=f\left(V_{\mathrm{e}}\right)$ où $V_{\mathrm{e}}$ est le volume d'élution, conduit à une loi de calibration universelle plus linéaire que celles obtenues jusqu'à présent [11], notamment dans le domaine des grandes et des petites masses moléculaires.

Remerciements. - Je remercie G. Weill pour une discussion fructueuse sur ce sujet.

\section{Bibliographie}

[1] Kurata, M., Yamakawa, H., J. Chem. Phys. 29 (1958) 311.

[2] Des Cloizeaux, J., J. Physique Colloq. 39 (1978) C2-135.

[3] Cotton, J. P., Decker, D., Farnoux, B., Jannink, G., OBer, R. et Picot, C., Phys. Rev. Lett. 32 (1974) 1170.

[4] Daoud, M., Jannink, G., J. Physique 39 (1978) 331.

[5] Weill, G., Des Cloizeaux, J., J. Physique (à paraître). Noter que dans cet article, $N$ est le nombre de segments statistiques.

[6] Mukherjea, R. N., Rempp, P., J. Chim. Phys. 56 (1959) 94.
[7] Freund, L., Thèse, Université de Strasbourg (1961).

[8] Delsanti, M., Thèse, Université Paris-Sud, Orsay (1978).

[9] Daoud, M., Cotton, J. P., Farnoux, B., Jannink, G., Sarma, G., Benort, H., Duplessix, R., Picot, C. et DE GeNNES, P. G., Macromolecules 8 (1975) 804.

[10] Farnoux, B., Boú́, F., Cotton, J. P., Daoud, M., Jannink, G., Nierlich, M. and De Gennes, P. G., J. Physique 39 (1978) 77.

[11] Gallot, Z., Thèse, Université Pasteur de Strasbourg (1970). 